\title{
A case of severe hypercalcemia in a young patient on the Neurorehabilitation Unit
}

Ahmad Shiraz, Roger Clark, Simon Howell, Kalpana Kaushal

Department of Endocrinology and Diabetes, Lancashire Teaching Hospitals Foundation Trust, Sharoe Green Lane, Preston, Lancashire.

\section{Introduction}

- Immobilization hypercalcemia ( IH ) typically develops 4-6 weeks post trauma. However it can present as early as two weeks and as late as six months.

- It is more common in children and adolescents due to an increased rate of bone turnover.

- We report a patient who developed hypercalcemia after sustaining multiple fractures and following prolonged immobilization.

\section{Case}

- A 24 year old man was admitted for neurorehabilitation after a road traffic accident, sustaining a subarachanoid haemorrhage and multiple fractures.

- Four months after admission, he developed hypercalcemia (3.63 $\mathrm{mmol} / \mathrm{l}$ ) and PTH was suppressed at $0.9 \mathrm{pmol} / \mathrm{l}$.

- He had mild renal impairment initially.

- Thyroid and adrenal function, serum ACE, Vitamin D level and PTHrp were normal. Myeloma was excluded. CT Thorax, abdomen \& pelvis and bone scan were unremarkable except for extensive heterotopic ossification around both hips (Figure 1). Heterotropic ossification is frequently seen with musculoskeletal trauma ${ }^{1}$ but it is not associated with pathophysiology of $\mathrm{IH}$.

- Normocalcaemia was achieved following intravenous fluids and zoledronic acid (Figure 2).

- After extensive investigation a diagnosis of $\mathrm{IH}$ was made. Serum calcium was normal when last checked 9 weeks after intravenous bisphosphonate therapy.

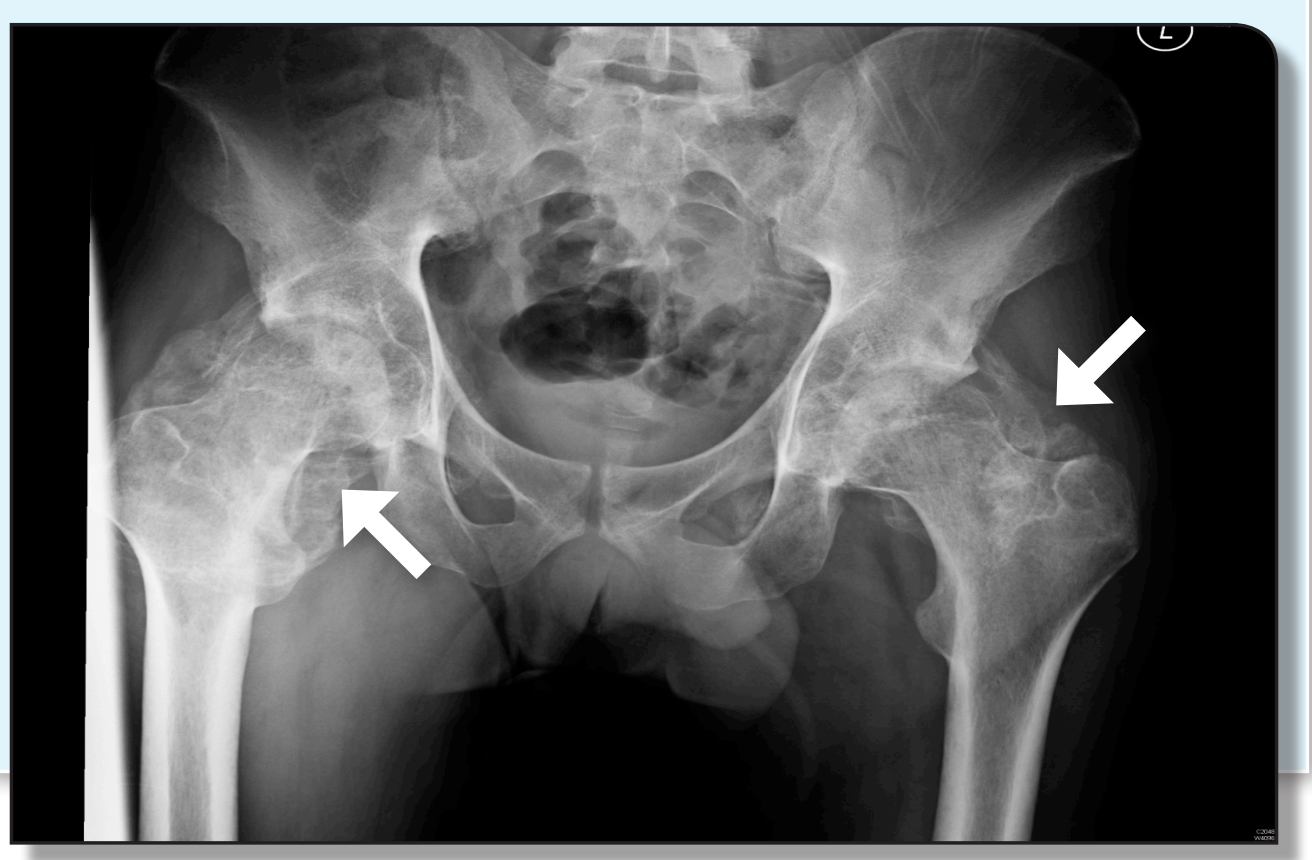

Figure 1 : Plain X-Ray Pelvis shows Heterotopic Ossification .

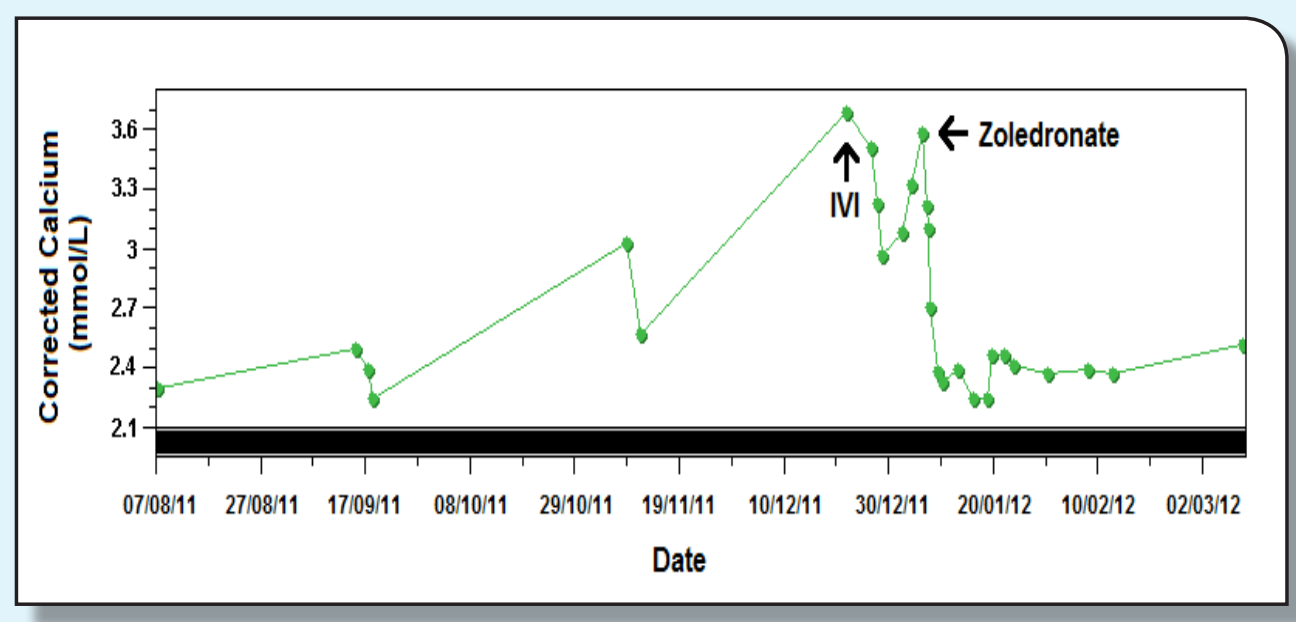

Figure 2: Serum calcium levels before and after intravenous bisphosphonate therapy.

\section{Discussion}

- $\quad \mathrm{IH}$ is an under-recognized cause of hypercalcaemia.

- In one study, it occurs in approximately $10-23 \%$ of patients with spinal cord injuries ${ }^{2}$.

- Immobilization is usually secondary to spinal cord injury, orthopaedic fracture, Guillain-Barre syndrome and cerebral vascular accident.

- The exact mechanism of $\mathrm{IH}$ is not clear. HM Frost ${ }^{3}$ described the mechanostat theory and proposed that $\mathrm{IH}$ is related to loss of mechanical stress on the bone, leading to increased osteoclastic bone resorption and reduced osteoblastic bone formation.

- Risk factors for IH include more severe immobilisation, pre-existing renal disease, childhood/adolescence, and metabolic acidosis.

- Passive mobilisation or weight-bearing rehabilitation appears to be effective therapy and should be instituted early.

- The majority of patients respond to a single dose of bisphosphonate. Occasionally a second dose is required. ${ }^{4}$

- Although our patient presented later after his trauma than average, the diagnosis should be considered in the appropriate clinical setting after excluding other common causes of hypercalcaemia.

- Clinical suspicion of IH may reduce the need for unnecessary invasive procedures.

References

Shehab D, Elgazzar AH, Collier BD. Heterotropic ossification. Department of Medicine, Faculty of Medicine, Kuwait University, Safat, Kuwait. J Nucl Med 2002 Mar 43(3): 346-53

Maynard FM : Immobilization hypercalcemia following spinal cord injury. Arch Phys Med Rehabil. Jan 1986;67(1):41-4.

Frost HM: Skeletal structural adaptations to mechanical usage (SATMU): 1. Redefining Wolff's Law: the bone modelling problem. Anat. Rec. 226, pp 403-413, 1990a.

Teresa L. Massagli , Diana D . Cardenas : Immobilization hypercalcemia treatment with pamidronate disodium after spinal cord injury. Archives of Physical Medicine and rehabilitation Volume 80, Issue 9 September 1999, Pages 998-1000. 